\title{
Part 3: Abstracts From the NCCN 19th Annual Conference: Advancing the Standard of Cancer Care ${ }^{\mathrm{TM}}$
}

The following abstracts were accepted for presentation at the NCCN 19th Annual Conference: Advancing the Standard of Cancer Care $^{\mathrm{TM}}$ General Poster Session at the Westin Diplomat in Hollywood, Florida, on March 13-14, 2014. NCCN considered original abstracts from investigators in the oncology community related to Best Practices in Implementation and Use of Clinical Practice Guidelines, Quality Improvement, Outcomes and Health Services Research, and NCCN Oncology Research Program-funded Projects.

\section{Outcomes and Health Services Research \\ AB2014.24. National Estimates of Health Care Services Expenditures for Patients With Prostate Cancer: An Analysis of the Medical Expenditure Panel Survey (2006-2010) \\ Palak Patel, MS, and Surbhi Shah, MS \\ The University of Georgia}

Background: Prostate cancer continues to represent a major source of medical expenditure. As the health care delivery costs continue to increase along with the rates of diagnosis and survival, increased attention must be paid to the patient's expenditure. The main purpose of this study is to analyze trends in total expenditure in patients with prostate cancer from 2006 to 2010. Because prostate cancer is highly prevalent in black men, the secondary objective of this study is to evaluate the total expenditure in prostate cancer based on race.

Methods: A longitudinal retrospective database analysis was performed from 2006 to 2010 using Medical Expenditure Panel Survey data. Various files, such as medical condition; prescription drugs; outpatient, inpatient, and emergency visits; office-based and home health care; and full-year consolidated files were combined and analyzed when patients with prostate cancer were identified using Clinical Classification Code 29. Descriptive statistics were used to assess trends, whereas domain analysis was used to estimate expenditure based on race. Results: Total expenditure in patients with prostate cancer was found to be approximately $\$ 3.7$ billion in 2006 , followed by approximately $\$ 12.0$ billion in 2007, $\$ 5.7$ billion in $2008, \$ 7.4$ billion in 2009 , and nearly $\$ 5.6$ billion in 2010. Thus, the expenditure was inconsistently reduced after 2007. Results of domain analyses showed that, on average, white patients spent more on health care than black patients in all years except 2007. Conclusions: Prostate cancer expenditure was found to be higher in 2007 . The literature has reported that overuse of health care services among patients with prostate cancer is a major concern among policy makers. The decreased expenditure after 2007 seen in this study may be attributed to low overuse of prostate cancer-related services. Lower expenditure may also be explained by use of observational treatment strategies, such as active surveillance in clinical practice, which has been the most recommended therapeutic option for men with low-risk prostate cancer by various organizations.

\section{AB2014.25. Emergency Department Use by Oncology Patients: A Systematic Review \\ Rebecca Salisbury, MSN, MPP; Janice Bell, MN, MPH, PhD; Hermine Poghosyan, PhD; Richard Bold, MD; Sarah Reed, MPH, MSW; and Jill Joseph, MD, PhD \\ University of California, Davis}

Background: Recent national reports call for interventions to reduce costly and potentially avoidable services, such as emergency department (ED) visits. ED use by oncology patients is appropriate for acute health concerns but is a poor alternative to adequately managed care and treatment regimens. Successful interventions to reduce avoidable ED visits by oncology patients hinge on understanding the burden of ED visits in this population. This systematic review examines the incidence and predictors of ED use by oncology patients.

Methods: A key word search was performed on the PubMed, CINAHL, and EconLit databases and the online bibliography of the SEER program. English peerreviewed research studies published between April 1, 2003, through March 31, 2013, were included if they had a sample size of more than 50 patients and measured the incidence or predictors of ED use among patients with cancer. Studies were excluded if they focused only on end-of-life care. All references cited in the included articles or related review papers were also examined.

Results: Of the 1048 articles identified, 892 were excluded based on review of titles and abstracts, and 140 
were excluded based on full text review; 16 studies were retained for data abstraction. These studies varied greatly in study aim, population of interest, and sample size. Thirteen were conducted in the United States and 3 were international. Twelve used a retrospective cohort design, and 4 analyzed data from prospective cohorts. Nine studies were populationbased, 6 were single-center studies, and 1 was a multicenter study. Eight focused on ED use among patients with one specific cancer type; 2 considered all cancers. Sample sizes range from 60 to more than 60,000 participants. The proportion of the study sample that visited the ED ranged from 1\% to $69 \%$. Some of this variation is attributable to differences in cancer diagnoses and in the time frame in which ED use was examined across studies (which ranged from 7 days to 1 year). Thirteen variables were identified as significant predictors of increased ED use, only 5 of which (African American race, older age, male sex, lower income, and symptom severity) were identified in more than one study.

Conclusions: Extant studies use different designs and examine different populations (by cancer type, treatment, and health insurance status). Collectively, the studies lack population-based estimates for all cancers combined, and therefore the full extent of ED use by oncology patients remains unclear. From a public health perspective, this body of evidence would be strengthened by use of comparable standardized definitions and methodologies to analyze recent population-based data for all cancer patients.

\section{AB2014.26. Computerized Relational Databases for Pediatric Non-Hodgkin's Lymphoma Using Microsoft SQL Server: A Useful Tool for Outcome Analysis and Research in a Cancer Treatment Hospital}

Khawar Siddiqui, MSc; Amani Al-Kofide, MD; Rafat Jafri, MBBS; and Asim Belgaumi, MD

King Faisal Specialist Hospital and Research Centre, Riyadh, Saudi Arabia

Background: Cancer is the second leading cause of death among children aged 5 to 14 years. The subspecialty of pediatric oncology cannot be graded on par with international standards of care without a fully functional set of clinical databases capable of abstraction, collation, collection, and management of diagnostic-, treatment-, and outcome-related data on the patients it treats.

Methods: The Department of Pediatric Hematology/Oncology at King Faisal Specialists Hospital and Research Centre, Riyadh, Saudi Arabia, registers an average of 280 pediatric patients with newly diagnosed neoplasms every year; approximately 15 of these are non-Hodgkin's Lymphoma (NHL). The increasing informational needs of the institution prompted the development of an in-house comprehensive, robust, and scalable database for these patients. Using the industry standard $\mathrm{Mi}$ crosoft SQL Server 2000 at the backend, relational databases were developed for NHL, with the data items decided by the team of treating oncologists based on demographics, diagnostics, treatment, infectious and noninfectious toxicities, and outcomes. The front-end of the database (e-CRF) was created using MS Access 2003. The database itself is linked with the other oncologyrelated databases, including the core database of minimum data set, relapse reporting, salvage therapy, bone marrow transplant, and expiry event for each patient. Data entry checks were incorporated both at the server level and at the front-end. Keeping in mind the clinical research study/trial requirements, handles were created to link this database with the same system managing the research subject enrollment, allowing it to record patient accrual and drop-out in various active research studies.

Results: The relational database, which was activated on January 1, 2005, now contains data on 135 cases using more than 40 tables. Running without any downtime and linked with the authors' existing bone marrow transplant data management system, it has played a vital role the timely provision of more than 10 data sets to the oncologists for their information needs relating to patient care, outcome analysis, and research. Accommodating the user needs in the system after deployment was almost immediate and was associated with no additional cost or undue downtime.

Conclusions: The development of an in-house, robust, and scalable system of data and information management using a relational database management system for pediatric patients with NHL is possible using commonly available resources in cancer treatment facilities. This system has also proved to be better than or on par with other commercially available solutions in terms of its development and maintenance cost. 
Clinical Oncology Research Trials

AB2014.27. Long-Term Follow-up of a Pivotal Phase II Study of Ultratrace lobenguane I-131 in Patients With Malignant Relapsed/Refractory Pheochromocytoma (pheo)/Paraganglioma (para)

Daniel Alexander Pryma, MDa; Daniel C. Sullivan, MD'; Camilo Jimenez, MD; ; Julie K. Schwarz, MD, PhDd; Richard B. Noto, MDe; Thomas Armor, CNMT; John Babich, MS, PhD'; and Robert J, Israel, $\mathrm{MD}^{\dagger}$

${ }^{a}$ Hospital of the University of Pennsylvania; ' $D$ Duke University Medical Center; 'The University of Texas MD Anderson Cancer Center;

${ }^{a}$ Washington University School of Medicine; ' Rhode Island Hospital; ${ }^{f}$ Progenics Pharmaceuticals, Inc; and ${ }^{g}$ Weill Cornell Medical College

Background: Ultratrace Iobenguane I-131 (Azedra) is a radiopharmaceutical under development for the treatment of patients with iobenguane-avid relapsed/ refractory malignant pheochromocytoma (pheo)/ paraganglioma (para). Produced using the proprietary Ultratrace platform, Azedra is designed to minimize unlabeled iobenguane being carried through from the production reaction to the final formulation, resulting in higher specific activity. This may result in a high cellular uptake of radioactivity and hence greater tumor uptake and reduced toxicity. The purpose of this analysis is to assess the long-term survival with up to 5 years of follow-up of patients treated in a phase II study of Azedra in malignant pheo/para.

Methods: Eligible patients were aged 12 years and older with a confirmed diagnosis of malignant pheo or para who were ineligible for curative surgery, and were on stable antihypertensive medication for tumor-related hypertension for at least 30 days before the first therapeutic administration. Patients initially received 3 to $6 \mathrm{mCi}$ of Azedra (the imaging dose) followed by 3 whole-body scans to confirm uptake of the study agent by at least 1 known tumor, assess biodistribution, and determine whole-body and organ dosimetry. Patients then received up to 2 therapeutic administrations of $8 \mathrm{mCi} / \mathrm{kg}$ up to a maximum of $500 \mathrm{mCi}$, each given 3 to 6 months apart. The primary end point was the proportion of patients with at least a $50 \%$ reduction of all antihypertensive medications lasting at least 6 months. Titrations of antihypertensive medications were made according to a standard algorithm. Statistical analysis was performed to determine whether the lower bound of the $95 \% \mathrm{CI}$ for the proportion of patients meeting the primary end point exceeded $10 \%$.

Results: A total of 49 patients were enrolled; 44 (26 men; 18 women) received the imaging administration, and 41 received at least one therapeutic administration. The primary end point was met by 13 patients
(31.7\%; 95\% CI, 16.2\%-47.2\%). All 41 patients who received at least 1 therapeutic administration experienced at least 1 treatment-emergent adverse event. Gastrointestinal and hematologic disorders were most frequently reported, and $29 \%$ of the patients experienced a serious adverse event of grade 3 or higher. To date, 18 deaths have occurred during the study, with 13 from disease progression; 10 patients remain in long-term follow-up. Overall survival was $93 \%$ and $69 \%$ at 12 and 24 months, respectively.

Conclusions: The overall results of this study support a dosing regimen of 2 doses of $500 \mathrm{mCi}$ of Azedra separated by 3 to 6 months. Thirteen patients $(31.7 \%)$ met the primary end point. In this study, Azedra was an effective and well-tolerated treatment for patients with iobenguane-avid metastatic and/or recurrent pheo/para, an orphan disease with no approved therapies.

\section{Neuro-Oncology, Clinical Practice \\ AB2014.28. Guidelines on the Use of Polymeric Chemotherapy in the Management of Glioblastoma: Meta-Analysis of Survival Outcomes and Safety \\ Timothy Ryken, MD, MSa; Sajeel Chowdhary, MD ${ }^{\text {b; }}$ and Herbert B. Newton, MD \\ aDepartment of Neurological Surgery, lowa Spine and Brain Institute; ${ }^{b}$ Department of Neuro-Oncology, Florida Hospital Cancer Institute; and 'Departments of Neurology, Neurosurgery, and Oncology, Wexner Medical Center at the Ohio State University and James Cancer Hospital}

Background: Carmustine wafers are an implantable polymeric chemotherapy approved by the FDA as a therapeutic adjunct in the treatment of both newly diagnosed and recurrent glioblastoma, and are incorporated in both NCCN care pathways and nationally approved practice parameters. Despite these recommendations, widespread use has been limited for a variety of reasons, including concern that the use of Gliadel has been an exclusion criterion for clinical trials. A meta-analysis was performed to clarify the impact of Gliadel on survival and safety to assist future investigators in planning more inclusive clinical trials.

Methods: This meta-analysis estimated survival times for patients treated with Gliadel for new or recurrent glioblastoma. Overall survival rates, median survival time, and incidence of safety outcomes were summarized. Median survival was compared between Gliadel- and non-Gliadel-treated patients using a 2-way analysis of variance.

Results: Sixty studies contributed to the analysis (Gliadel: $n=3162$; non-Gliadel: $n=1736)$. Among newly diagnosed Gliadel-treated patients, the 1- and 2-year 
survival rates were $66 \%$ and $26 \%$ versus $41 \%$ and $16 \%$, respectively, for non-Gliadel-treated patients. Median survival times were $16.1 \pm 21.7$ months for newly diagnosed Gliadel-treated patients versus $12.8 \pm 28.9$ months for newly diagnosed non-Gliadel-treated patients. Significant effects of treatment $(P=.023)$ and diagnosis $(P<.001)$ were detected, with no significant interaction between them $(P=.268)$. The most common adverse event associated with wafer removal was surgical site infection; the most common adverse events for repeat surgery were mass effect, surgical site infection, hydrocephalus, cysts in resection cavity, hematoma, wound healing complications, and brain necrosis.

Conclusions: The exclusion of patients from clinical trials based on the use of Gliadel, an FDA-approved therapy also incorporated into NCCN care pathways, is concerning. The hope is that through more clearly defining the impact of Gliadel, future investigators will choose to plan more inclusive clinical trials for new and recurrent glioblastoma.

\section{Hereditary Cancer Syndromes}

AB2014.29. Evaluating the Personal and Family History Overlap Between Hereditary Cancer Syndromes

Jennifer Saam, LCGC, PhD; Chris Arnell, MBA;

Kelsey Moyes, MStat; Kirstin M. Roundy, MS;

Ingrid Marino, MS, CGC; and Richard J. Wenstrup, MD

Myriad Genetic Laboratories, Inc.

Background: Current NCCN Guidelines for hereditary cancer genetic testing are divided among different cancer site guidelines. Guidelines for colorectal cancer screening cover several hereditary colon cancer syndromes (Lynch syndrome [LS], familial adenomatous polyposis [FAP], MUTYH-associated polyposis, Peutz-Jeghers, and juvenile polyposis), whereas guidelines for hereditary breast cancer testing include hereditary breast and ovarian cancer (HBOC) and Li-Fraumeni and Cowden syndromes. The premise underlying this system is that hereditary cancer syndromes have distinct cancer profiles. However, overlapping cancers among these syndromes make it difficult to identify which diagnosis is most appropriate for a given patient. To quantify the overlap among hereditary cancer syndromes, the personal and family history of patients in a genetic testing population was reviewed. Results from hereditary cancer panel testing were also reviewed to assess the potential benefit of this technology for patients with overlapping cancer phenotypes.
Methods: Personal and family histories submitted with genetic testing for LS or HBOC were reviewed to identify patients who met testing criteria for both syndromes at a commercial laboratory. Tests for a specific family mutation, the common Ashkenazi Jewish HBOC mutations or a single LS gene, were excluded. The commercial testing results of a 25-gene hereditary cancer panel were also reviewed to identify patients who were tested because of the clinical presentation of one syndrome but had a mutation associated with the other.

Results: Of patients tested for HBOC, $8 \%$ had family histories that met $2012 \mathrm{NCCN}$ criteria for LS. Of patients tested for LS, 30\% met criteria for HBOC, showing extensive overlap between these syndromes. Of the patients with $\mathrm{HBOC}$ whose criteria overlapped with those in the LS guidelines, $89 \%$ qualified based on family history and $10 \%$ qualified based on personal cancer history. Of patients with LS who also met criteria in the HBOC guidelines, 59\% qualified based on family history, $19.4 \%$ qualified based on personal cancer history, and $20.3 \%$ qualified based on a combination of personal and family history. Panel testing identified a BRCA2 mutation in a patient with early-onset colon cancer and a family history of colon and pancreatic cancer. Panel testing also identified a patient with early-onset breast cancer with an MSH6 mutation. Neither of these patients met NCCN criteria for the syndrome associated with these mutations.

Conclusions: This analysis suggests that genetic testing strategies that focus on a single hereditary cancer syndrome may miss patients with genetic syndromes because of complicated cancer histories. Advancements in sequencing technology, including hereditary cancer panels, will allow patients to receive the appropriate diagnosis and will require new guidelines to be developed as this technology becomes part of the standard of care.

Best Practices in Implementation and Use of Clinical Practice Guidelines

\section{AB2014.30. An Analysis of NCCN Guideline Development and Transparency Processes Through the Use of Panel Meeting Minutes}

Iris Tam, PharmD; ; Tien Ho, PharmD Candidatea, ${ }^{\mathrm{a} b}$; and Anh Doan, PharmD Candidate ${ }^{a, b}$

${ }^{a}$ Genentech Inc; ${ }^{b}$ University of California at San Francisco School of Pharmacy

Background: NCCN develops a set of clinical practice guidelines that have become influential in establishing 
standards of care in oncology. Individual panels post the meeting minutes for public view on the NCCN Web site after discussions on requests for changes from internal and external sources. The purpose of this review is to increase user understanding of the NCCN Guidelines development and transparency processes through an extensive analysis of panel meeting minutes.

Methods: Panel meeting minutes posted on the NCCN Web site (NCCN.org) were last accessed on October 30, 2013. The following data were extracted by an individual reviewer: panel, date, request for change, source of request, panel decision, and references cited. Data collection was validated by a second reviewer. References cited by NCCN Panels were found on PubMed and the level of evidence was categorized.

Results: Between April 2010 and September 2013, NCCN panels had 411 discussions on requests for changes to the guidelines. Panels with the highest number of discussions ( $>8 \%$ ) were those for non-Hodgkin's lymphoma, non-small cell lung cancer/malignant pleural mesothelioma/thymomas, and colon/rectal/anal cancers. Sources of the requests were internal NCCN Member Institutions ( $77 \% ; n=318)$, external sources alone $(12 \% ; n=48)$, and concurrent internal and external sources $(11 \% ; n=45)$. The most common requests for changes to the guidelines $(>10 \%)$ were to add a regimen $(27 \%)$, add a drug (25\%), review data (17\%), and review treatment recommendations (11\%). Most of requests $(94 \% ; n=385)$ for change resulted in an update to the guidelines. Of the 18 requests that led to no update, the most common reason was lack of data (50\%). The average number of references cited per decision was 1 to 2 . Twenty-eight percent (28\%) of panel decisions to update the guidelines did not specify the references used. The highest level of evidence used to support the NCCN Panel decisions was based on phase II (32.6\%) or III data $(44.5 \%)$. The publication statuses of these references were either full journal article (69\%) or abstract/poster (29\%).

Conclusions: NCCN Panel meeting minutes from 2010 to 2013 reveal that most requests for updates to guidelines are received from NCCN Member Institutions. Adding a regimen or drug to the treatment recommendations constituted the most common type of request. Most requests result in an update to the guidelines, and the evidence used most by NCCN Panels to support the decision is based on phase II or III data. NCCN Panel meet- ing minutes, available for public view, provide key insights and improve transparency of the NCCN Guideline development process.

Shortened-Course Breast Cancer Conservation Radiotherapy

AB2014.31. Solid Modulated Accelerated Radiation

Therapy (SMART) for Early-Stage Breast Cancer

Theodore. E. Yaeger, MD

Wake Forest University School of Medicine, Caldwell Memorial Hospital

Background: Accelerated partial-breast irradiation is being studied as an alternative to whole-breast radiation for treating early-stage breast cancers. Currently 3 common methods are used: multicatheter brachytherapy, single-catheter brachytherapy, and external-beam limited-volume radiotherapy. The purpose of this study is to introduce a modification to standard external-beam techniques consistent with those ASTRO introduced as part of the "Choosing Wisely" campaign (available at: http://www.choosingwisely.org/astro-releases-list-of-five-radiationoncology-treatments-to-question-as-part-of-national-choosing-wisely-campaign/), which recommend considering a shorter course of radiotherapy when appropriate. This proposed regimen treats with intensity-modulated radiation therapy (IMRT), and allows concurrent partial-breast irradiation (seroma surgery site) and conventional whole-breast prophylaxis with a shortened course using a beam modulator (compensator) technique.

Methods: Patients with stage 0 or 1 or early stage 2 breast cancers were planned for both conventional tangent-to-boost and IMRT delivery. Patients who developed improved dose homogeneity with IMRT were then treated with the beam-modulated technique. Patients without any advantage from IMRT planning were treated conventionally using halfbeam blocked tangent fields followed by a seroma electron or photon boost. The populations were compared for demographics, treatment tolerance, cosmetic appearances, and cancer control outcomes.

Results: During 6 consecutive years of breast radiotherapy at a rural outreach cancer center, 73 patients were treated for breast conservation. The beam-modulated technique was the IMRT option. Subsequently, the 73 patients were evaluated using the criteria described previously; 35 patients had no improvement in their dosimetry plans and were treated conventionally, and 38 patients were offered IMRT. All 73 patients were judged for similar demographics, 
acute treatment tolerances, cosmetic outcomes (patient self-assessment and Harvard scale), and cancer control (local control and disease-free survival). To date, no patient in either arm has experienced a local, regional, or distant failure. However, nearly $50 \%$ of patients treated with the conventional technique had treatable acute skin reactions (grade 2 or 3 ), whereas only one in the IMRT group (grade 2) needed skin care. Furthermore, no patient in the IMRT group developed global breast edema or any cosmetic defect. Cosmesis was considered "acceptable" by patients in both groups. Harvard scale cosmesis was considered by professional staff to be generally "good to good+" in the tangent group and "good+ to excellent" in the IMRT group (a superior result) at follow-up at 1 and 6 months and at 1 year.
After acute reactions subsided, subsequent cosmesis remained stable for both groups.

Conclusions: Solid modulated accelerated radiotherapy (SMART) is a useful form of IMRT that yields similar tumor control probabilities to conventional radiation (tangent-to-boost) techniques. For patients with larger-volume tumors with improved planning dosimetry, SMART seems to significantly prevent acute patient treatment skin reactions and produces long-term improved cosmetic outcomes, including edema control. Consistent with the "Choosing Wisely" recommendations, SMART has proved to be a shortened curative radiation course and a noninvasive alternative for treating low regional risk early-stage breast cancer. 